\title{
Influence of design embodiment on the performance of squeeze-film levitation contacts
}

\author{
A. Almurshedi ${ }^{*}, 1$, M. Atherton ${ }^{1}$, C. Mares $^{1}$, T. Stolarski ${ }^{1}$, M. Miyatake $^{2}$
}

Abstract - Conveying of lightweight and delicate parts is an important issue in some manufacturing. Two levitation devices, one based on horn transducer (Langevin type) and the other one in the form of a plain rectangular plate made of aluminium firmly fastened at both ends with a surface-mounted piezoelectric actuator, are compared. Both devices operate on a squeeze-film levitation (SFL) mechanism. To this end, physical experiments were carried out and it was found that the device utilizing horn-type transducer yields better levitation performance.

Keywords: floating; levitation; piezoelectric; squeeze-film.

\section{Introduction}

Non-contact, oil-free, precise and low wear transport along a production line is desirable for enabling accurate industrial products in micro-fabrication and nanotechnology contexts, such as semiconductor silicon wafers, micro-components, integrated circuits, and precision positioning.

The literature reveals that levitation comprises three main categories, namely: Standing Wave Levitation (SWL), Near-Field Acoustic Levitation (NFAL) and Squeeze film levitation (SFL). SWL lifts tiny particle substances placed between two structures (reflector and radiator) due to the reflection of a standing pressure wave.

\footnotetext{
${ }^{1}$ College of Engineering, Design and Physical Sciences, Brunel University London, UK.

${ }^{2}$ Department of Mechanical Engineering, Tokyo University of Science, 1-3 Kagurazaka Shinjuku-ku Tokyo 162 8601 , Japan. *Corresponding author: +447448613754

E-mail address: 1331834@brunel.ac.uk
} 
In a near-field acoustic levitation (NFAL) system, there is no need for the reflector as it is represented by the floating item itself. NFAL is created in the clearance between a vibrating surface and the item to be floated and has the potential to lift heavier items than SWL.

The NFAL effect depends on acoustic radiation pressure and the speed of sound in the fluid domain. Squeezefilm levitation (SFL) relies on a similar working principle to NFAL, occurring when the object to be floated is located adjacent to a vibrating structure [1]. However, it is the viscosity and compressibility of a thin film of air (squeeze-film) that is trapped between the two surfaces (vibrating surface and floating object) that forms the basis of SFL rather than sound pressure.

The average pressure in the squeeze film of SFL will be greater than the surrounding atmospheric pressure to produce a lifting force. This method can be mathematically expressed in terms of the nonlinear Reynolds equation and equation of motion.

There is some confusion in the levitation literature as SFL is used interchangeably with NFAL [13, 17, 20]. In NFAL, pressure is considered to be produced by the sound velocity or acoustic field created by a driving surface [1]. In SFL it is held that a time- average pressure generated in the clearance between surfaces (driving and levitating) is greater than the ambient pressure. The theoretical approach of SFL is based on Bernoulli's Principle whereas NFAL is based on acoustic radiation and sound velocity in air [2, 3].

In this paper, devices tested operate on the SFL effect. SFL was first deliberated by Langlois, in 1961 [2], then the effect had been observed by several investigators and applied to different contactless prototype designs. Hashimoto et al [3] investigated the characteristics of near-field acoustic levitation method to evaluate floating stability. It was based on an ultrasonic transducer design and utilised its flexural oscillation to generate the levitation forces.

Matsuo et al [4] evaluated the performance of the separation force exerted on an object to be lifted, based on a NFAL approach. The study utilises a horn transducer as a levitation prototype design shows that the force vastly depends on the oscillation mode of the driving surface. Ueha et al [5] implemented contactless levitation for an object of kg scale through a prototype design made from transducers. Flexural traveling 
waves created were considered as an important influence for floating the object. Minikes and Bucher [6] observed theoretically and experimentally the dynamic characteristics of a floating object utilising a horn transducer design. The investigations were based on the squeeze-film approach and divided the working principle into spring and damper elements determined by the driving frequency, confirmed by the findings. Nomura et al [7] investigated NFAL theoretically and experimentally in order to understand its fundamental mechanism. The study used a horn transducer connected to different aluminium discs to observe the influence on the floating characteristics. Hu et al [8] studied the stability of a floating disc placed on a vibrating transducer design. The study evaluated NFAL. A floating design was utilised that comprised a horn transducer with a radiator plate and a reflector to produce NFAL [8]. Atherton et al [9] described the fundamental aspects of NFAL and SFL for practical bearing designs. Minikes et al [10] inspected analytically and numerically the flow made by oscillations normal to a flat surface method and showed that by flexural wave propagation parallel to the surface it can lead to contactless lift. The investigations based on a SFL. Moreover, an analytical solution for the levitation force was also obtained.

Stolarski and Woolliscroft [11] examined levitation features for lightweight objects placed on a vibrating plate which was excited by actuators glued underneath the plate. The study was based on the SFL phenomenon and it was found that the actuators position governed its effectiveness. Wang and Wei [12] studied the SFL generated by a mixed-modal disc excitation utilising a horn transducer design. The physical studies identified the modal shapes of the disc that produced levitation. Ilssar and Bucher [13] presented a simplified analytical model defining the main dynamics of NFAL floating items, through adapting the equation of motion and Reynold's equation into a compact equation in which the local stiffness and damping are recognisable. The study utilised a horn transducer design. Ilssar et al [14] carried out structural optimisation of a levitation design based on a horn transducer that aimed to improve efficiency and, with an accurate axial floating capability, to meet the high-precision requirements of the micro-electronics fabrication industry. The study analysis was based on the NFAL method. 
Investigation of a NFAL based device consisting of a vibrating plate excited by round piezoelectric actuators attached to the underside of the plate is described in [15]. It was found that plate modal and forced harmonic shapes were important determinants of levitation characteristics.

Wei et al [16] made a new type of acoustic levitation design utilising reverse hydrodynamic effects of a nonparallel squeeze film which is occurred because of asymmetric pressure distribution. The study used horn transducers and a plate to investigate the floating performance. Li et al [17] studied the effect of structural modification on levitation improvement, based on SFL utilising a horn transducer design. It was found that a pressure gradient was the source of tangential velocity and force to induce the levitation.

SFL is considered to be the best method of non-contact transportation, compared to others such as magnetic, optical (utilising visible laser light to create radiation pressure), and aerodynamic, as confirmed by the literature [18]. The advantages of SFL are firstly, it is suitable for lifting fragile or sensitive components down to micron-scale. Moreover, contamination and friction encountered with classical production equipment can be avoided. In this study, a comparison is made of two different prototype SFL designs to achieve a frictionless movement for lightweight objects placed on the designs. The main aim is to identify an effective non-contact movement design in terms of the design capability, complexity, and cost.

2. Tested devices and experimental methodology

\subsection{Tested devices}

One of the designs (Design A) that has been used in this study is called the ultrasonic chuck, for which an ultrasonic transducer (Langevin, FBI 28452 HS) comprises the main part. The other (Design B), is a vibrating plate design, which consists of a plain rectangular plate made of aluminium firmly fastened at both ends. Table 1 provides essential information regarding material mechanical properties and dimensions for both designs. In addition, four rounded piezoelectric actuators of size $28 \mathrm{~mm}$ diameter $\times 0.5 \mathrm{~mm}$ thickness are glued onto the underside of the plate (Fig.1). The vibrating plate is clamped at both ends in the horizontal plane through a steel supporting structure. In addition, the dynamics of the levitation of both designs (A and 
B) have been investigated based on SFL. The investigation includes physical measurements for the vibrating plate design through using a digital image correlation system (DIC).

TABLE 1

\begin{tabular}{c|ccc}
\multicolumn{5}{c}{ DESIGNS SIZES AND MATERIAL PROPERTIES } \\
\hline $\begin{array}{c}\text { Design No. } \\
\text { Material } \\
\text { Geometry } \\
\text { Size of plate } \\
\text { [mm] }\end{array}$ & $\begin{array}{c}\text { Aluminium } \\
\text { Circular }\end{array}$ & $\begin{array}{c}\text { Aluminium } \\
\text { Circular }\end{array}$ & $\begin{array}{c}\text { Aluminium } \\
\text { Rectangular }\end{array}$ \\
$\begin{array}{c}\text { Resonance } \\
\text { frequency [kHz] }\end{array}$ & $200 \times 1$ & $200 \times 2$ & $200 \times 100 \times 2$ \\
Young's & 25.8 & 26.4 & 0.4 \\
Modulus Y & 71 & 71 & 69 \\
$\quad[\mathrm{GPa}]$ \\
$\begin{array}{c}\text { Density } \rho[\mathrm{N} / \mathrm{m} 3] \\
\text { Poisson's Ratio v }\end{array}$ & 2676 & 2676 & 2600 \\
\hline
\end{tabular}

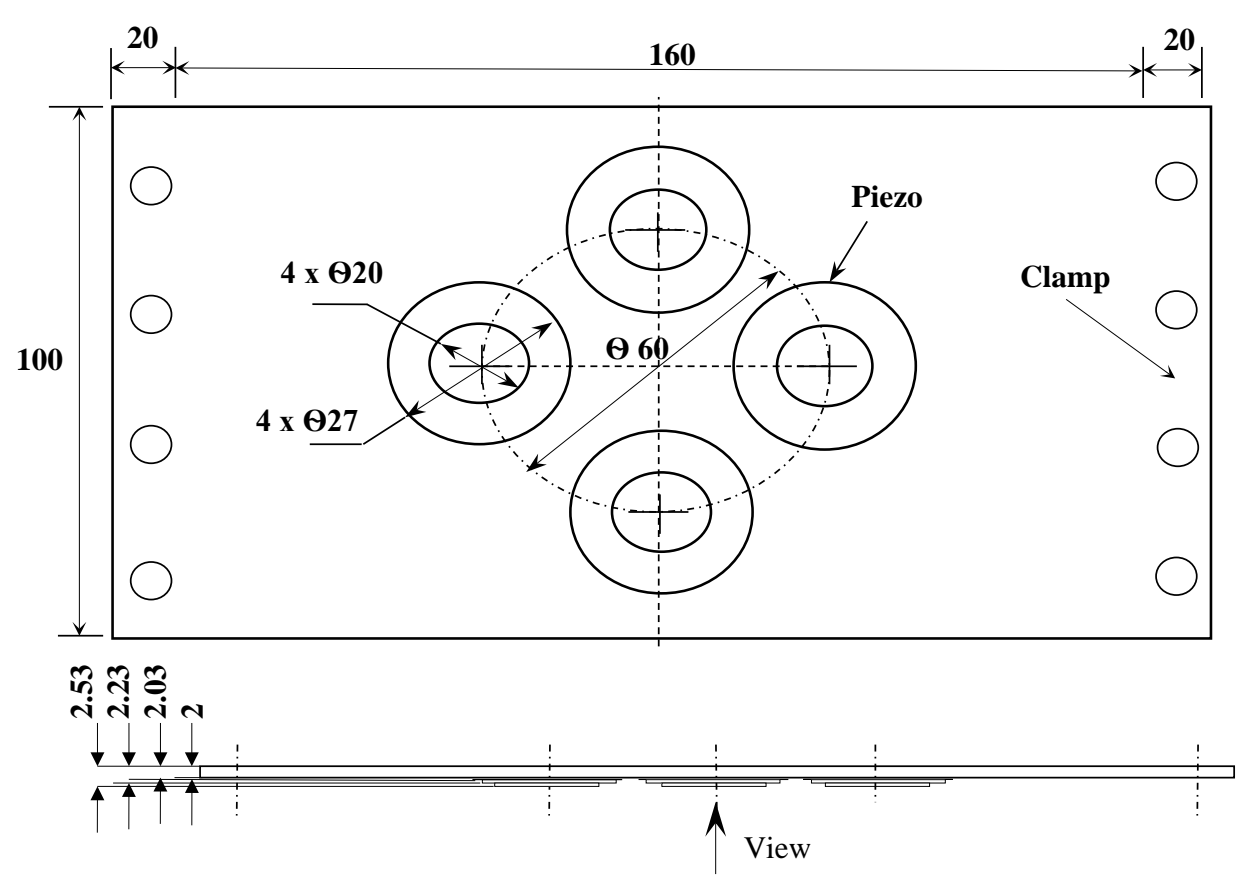

Fig. 1 CAD model of Design B (all dimensions in $\mathrm{mm}$ ). 


\subsubsection{Horn design (design A)}

Figure 2 shows, schematically, various horn's shapes frequently used in SFL devices. In this research, a conical shape was utilised and is shown in Figure 3. The horn is divided into a cylindrical part and one for amplifying the amplitude, with the length of the cylindrical portion being calculated from the driving frequency and the sound velocity of the longitudinal wave in the material. The cross-sectional area of the horn tip end is defined as the horn narrow end area $\left(S_{1}\right)$, the cross-sectional area where the cylindrical portion and the amplification portion are connected to the horn's thick end surface area $\left(S_{2}\right)$, the cross-sectional area of cylindrical portion is notified as $\left(S_{b}\right)$.

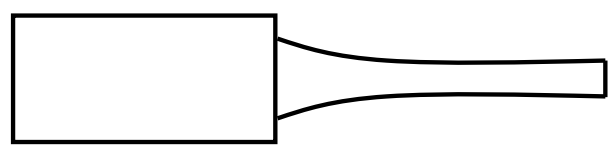

Exponential

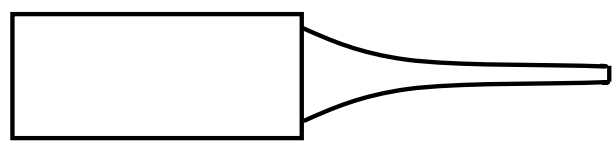

Catonoidal

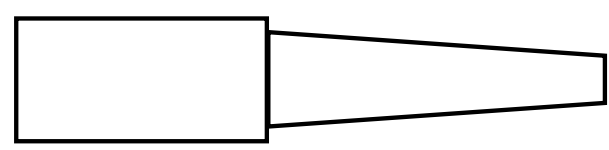

Conical

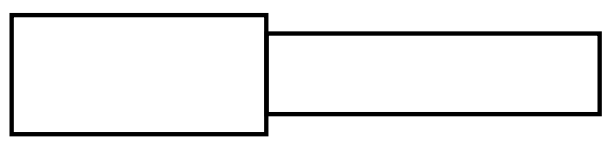

Uniform step

Fig. 2 Types of horn design

It is important when designing a horn, first, to determine the cylindrical part area from the transducer to be used and then, finding the thickness end area. If the area ratio with this case is made too large, the step portion becomes too discontinuous, and vibration propagation is obstructed. Experimentally, it should be less than 4:1 and in this experiment, this area ratio was 3: 1 in consideration of the balance of the experimental apparatus as a whole. Next, the horn's narrow end area is determined. The smaller the area is, the more the longitudinal wave vibration generated from the vibrator is amplified, but in this experiment, it is necessary to fasten the vibration plate to the tip of the horn by bolts. So, the tip diameter of the horn is set to 10 [mm]; $S_{2} / S_{1}=R_{2} / R_{1} ; R_{1}$ is the radius of the narrow face and $R_{2}$ is the radius of the wide face. Wave length constant can be expressed by $k=\omega / c$, in which $\omega$ is driving angular frequency and $c$ is sound velocity of the material. The full length of the horn can be represented by $L_{a}=\lambda / 4+L$, in which, $L$ is the amplification unit (Fig. 4). 


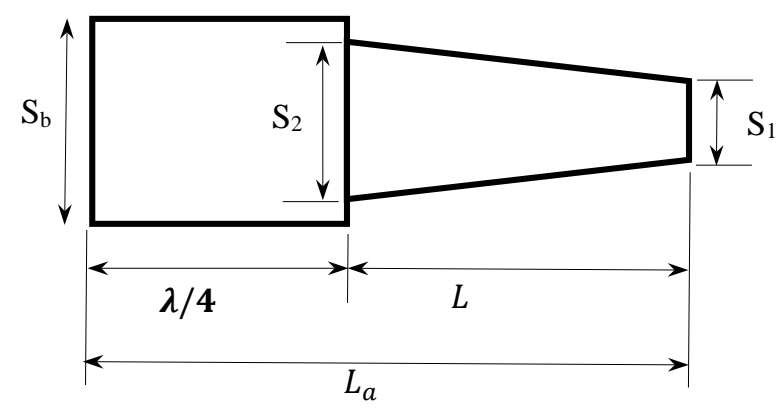

Fig. 3 Conical horn configuration.

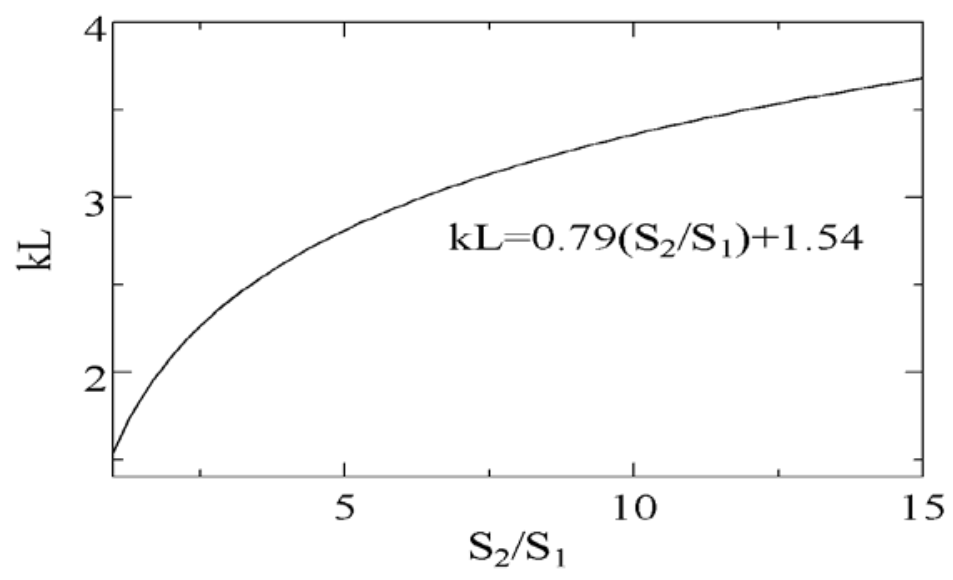

Fig. 4: Relationship between $S 2 / S 1$ and $k L$

The ultrasonic transducer used in this experiment, a ring-shaped piezoelectric element, is sandwiched between two metal blocks and tightened with a bolt to form an integral structure, which is also called a bolted Langevin type vibrator. A longitudinal wave (ultrasonic vibration) generated by an ultrasonic vibrator will transmit to a flat circular plate (dia. $200 \mathrm{~mm}$ ) attached to it and made of aluminium.

\subsubsection{Design A testing methodology}

Figure 5 show all essential elements of experimental testing applied to the design A. A sine wave voltage is an input to the ultrasonic vibrator, the vibration of which is amplified by the horn, and the vibration plate resonates at a certain natural frequency. This creates a squeeze air film and radiated pressure field between the oscillating plate and the object (supporting object) placed on the oscillating surface, whereby a repulsive force acts on the supporting object and then, this can float on the oscillating plate. The experimental equipment that can be used to operate Design A to achieve the floating and to measure its height is as follows. 
Firstly, an output voltage from a function generator is amplified to an arbitrary voltage via the actuator driver and input to the ultrasonic transducer. Secondly, the ultrasonic vibrator, vibrates by applying a sinusoidal voltage that gives a vibration displacement after providing a bias voltage, thereby creating a constant displacement. In addition, the amplitude voltage and frequency are measured throughout the process by an oscilloscope (Fig.5) and (Fig.6). Moreover, a non-contact displacement sensor (model: PHILTEC RC60 H3) (see Fig.6) is used for measuring the vibration amplitude of the deformed surface and the separation distance of the floating object. The sensor is connected to an oscilloscope, which displays the output signal in volts. The voltage corresponds to the dynamic distance of the circular plate and the levitating object's height. The measurements of the maximum levitation for three different semiconductor wafers of weights 52 $\mathrm{g}, 70 \mathrm{~g}$ and $88 \mathrm{~g}$ used as floating objects were taken. The vibration mode of the driving plate is visualized by scattering tiny plastic particles on the surface of the plate, while resonating it (Fig.7). 


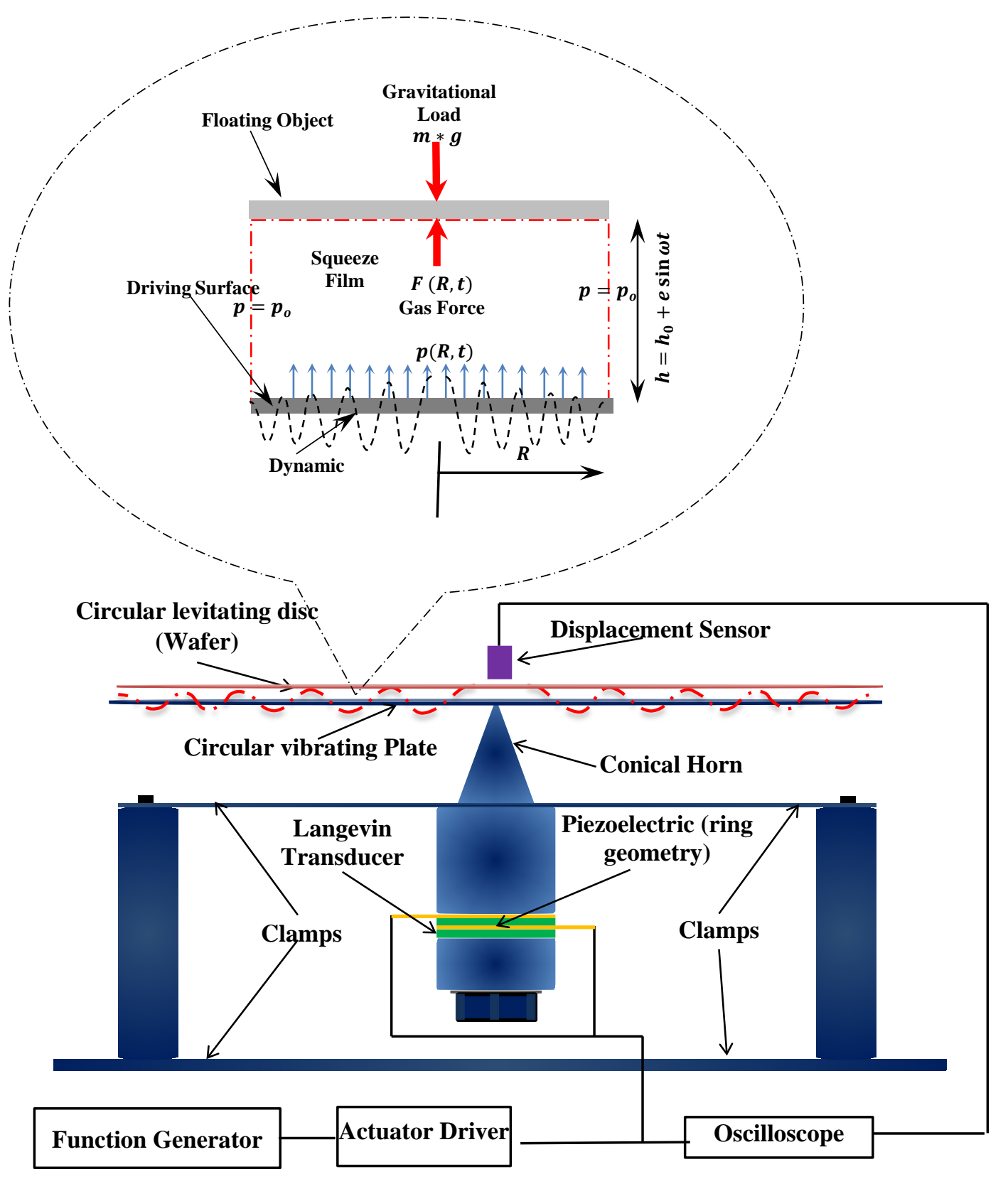

Fig.5 Experimental layout of SFL Design A 


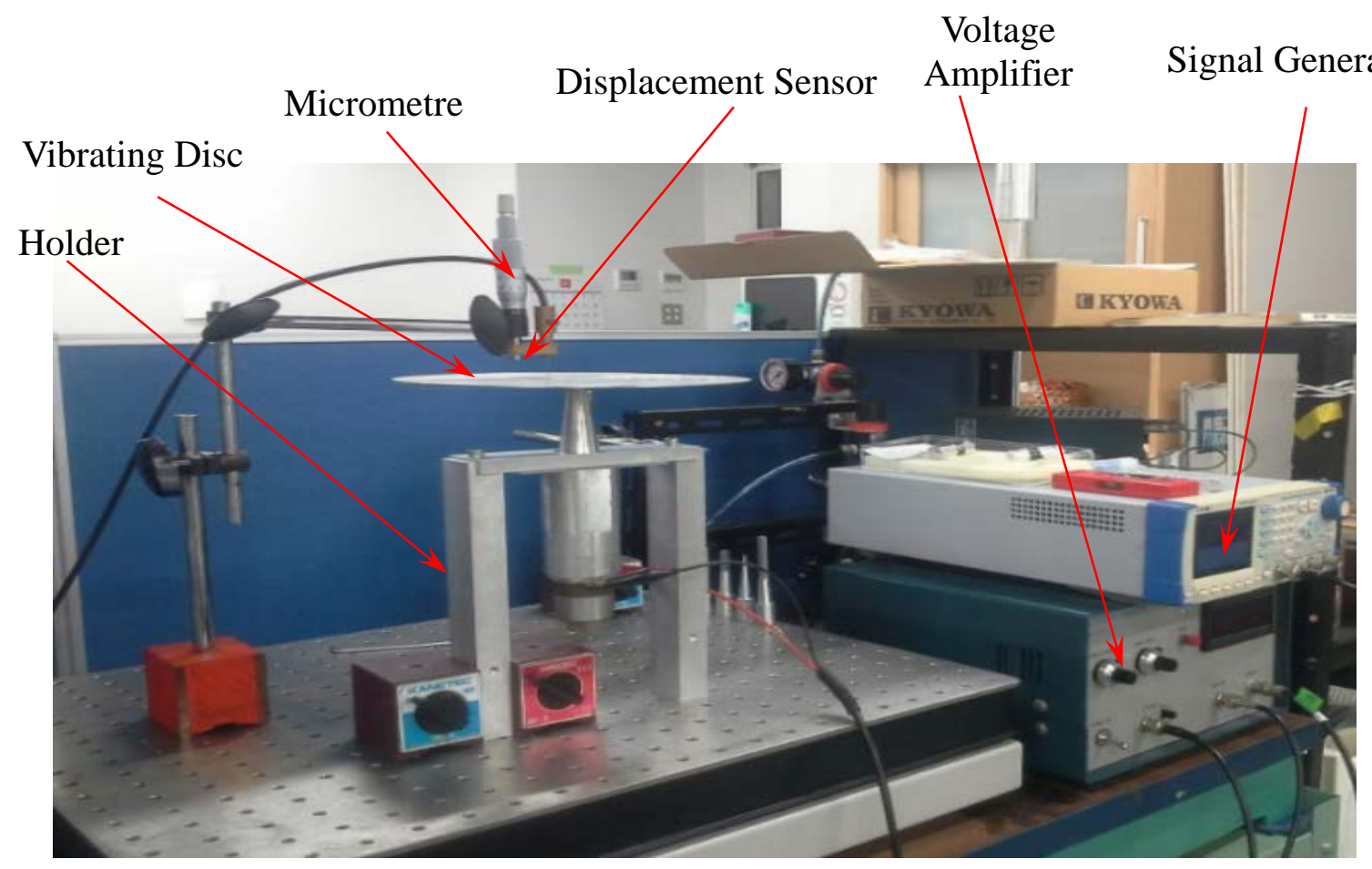

Fig.6 Experimental set-up of SFL Design A

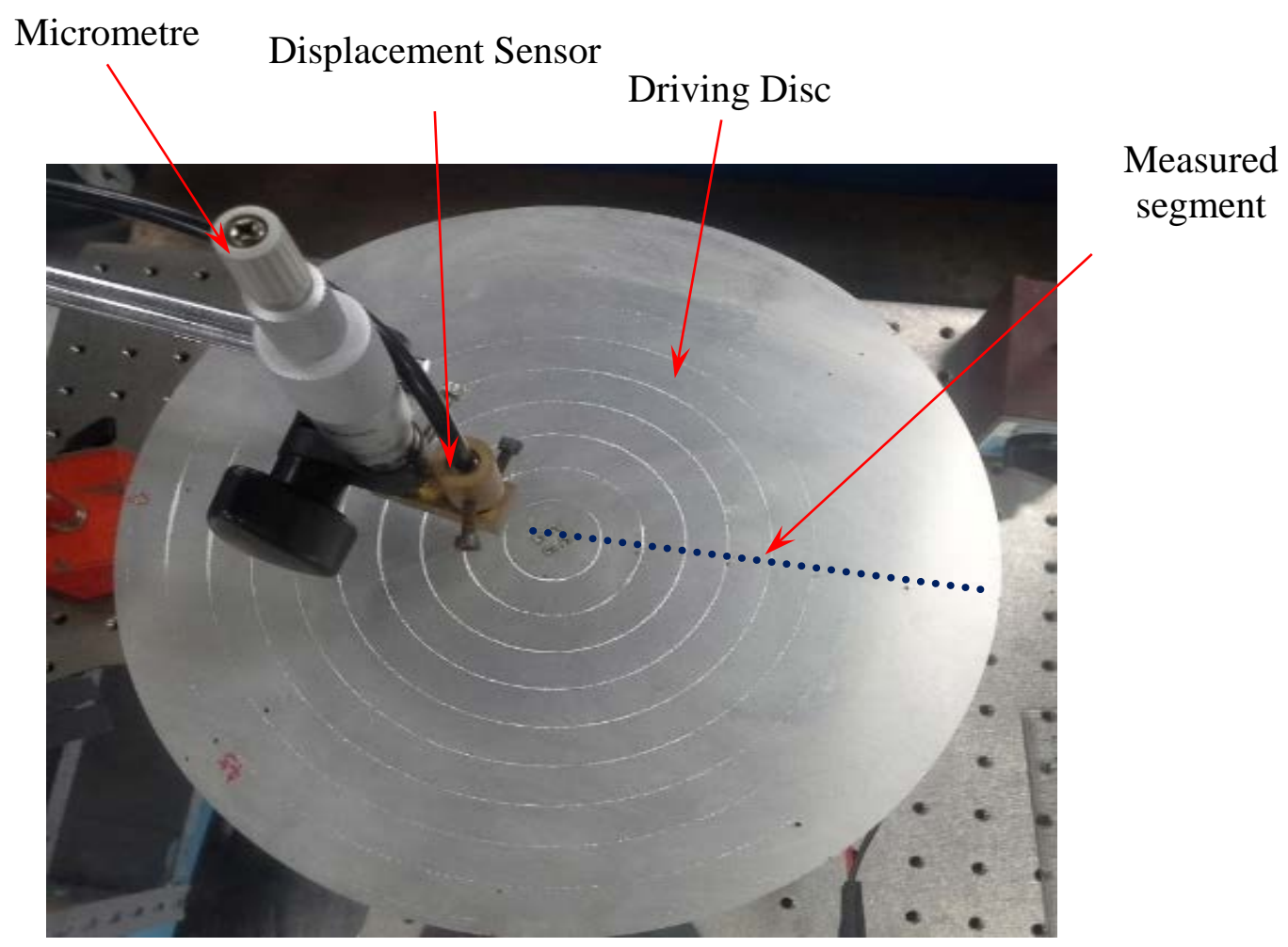

Fig. 7 Experimental oscillating plate Design A1 at $26.4 \mathrm{kHz}$. 


\subsubsection{Plate design (design B)}

The apparatus used for testing design B consists of a sine wave signal generator connected to an amplifier of type ENP-1-1U (Fig. 8) to magnify the sine wave voltage produced by the signal generator. The amplified voltage and sinusoidal frequency are supplied to drive the four piezoelectric actuators that are attached to the underside of the plate. The actuators are driven with an AC voltage, which produces a dynamic displacement on the top surface of the plate. The displacement will create a cyclic squeeze-film for the air trapped between the vibrating plate and the object. A decompression and compression process for the air film will suspend the object placed on the vibration plate. Digital image correlation (DIC) system was used to observe deformation modes of the plate.
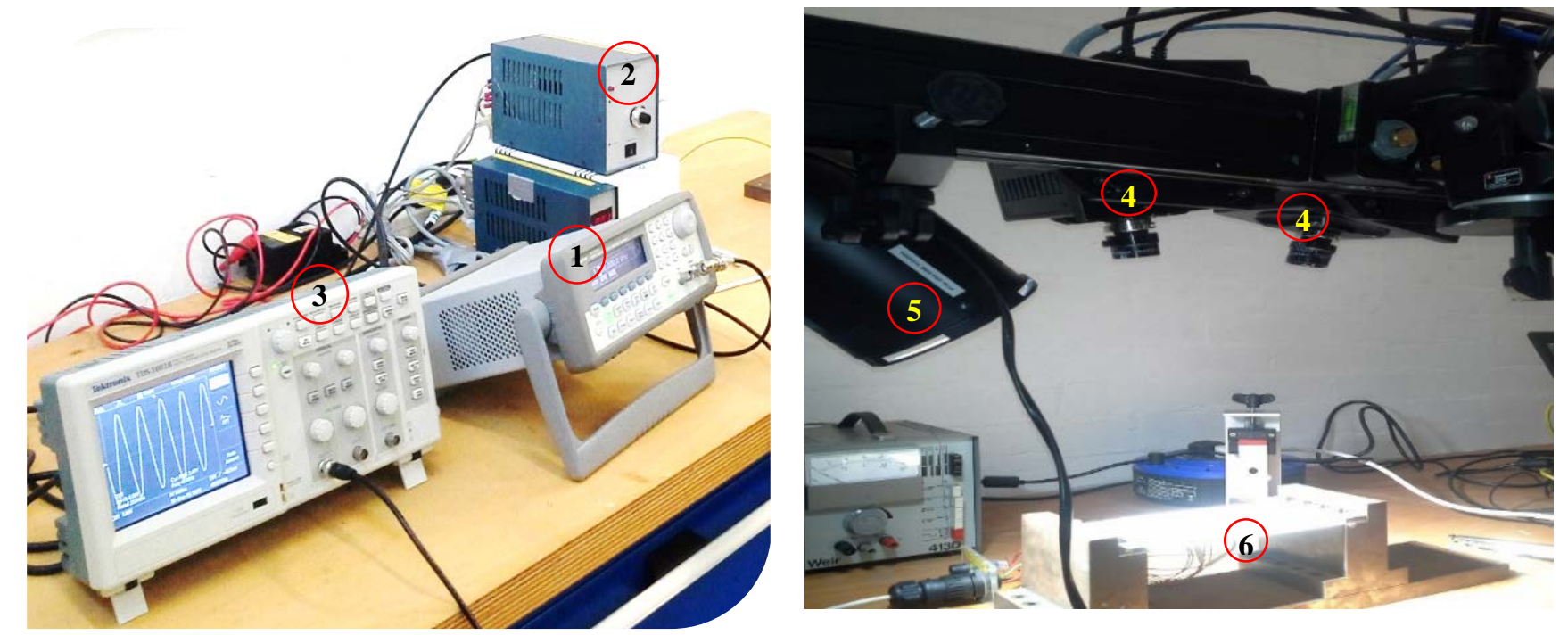

(1) Signal Generator.

(2) Voltage Amplifier.

(3) Oscilloscope.

(4) High speed camera (DIC system) (ARAMIS - Photron UX50).

(5) Light (DIC system).

(6) Vibrating plate design.

Fig. 8 Experimental apparatuses set-up (Design B). 


\section{Results and discussions}

\subsection{Design A}

Observing the pattern formed by small particles deposited on the surface of the disc (see Fig.7) one can figure out the nodes and anti-nodes in the disc's deformation. The results of measurements with the non-contact displacement sensor are shown in Figure 9 for the resonance frequency of $26.4 \mathrm{kHz}$ (design A1) while Figure 10 shows the measurements for design A2 and its resonance frequency of $25.8 \mathrm{kHz}$.

The sideways movement is avoided through four evenly placed cord restrictors with little clearance around the rig design in order to keep the wafer approximately centralised on the driving structure.

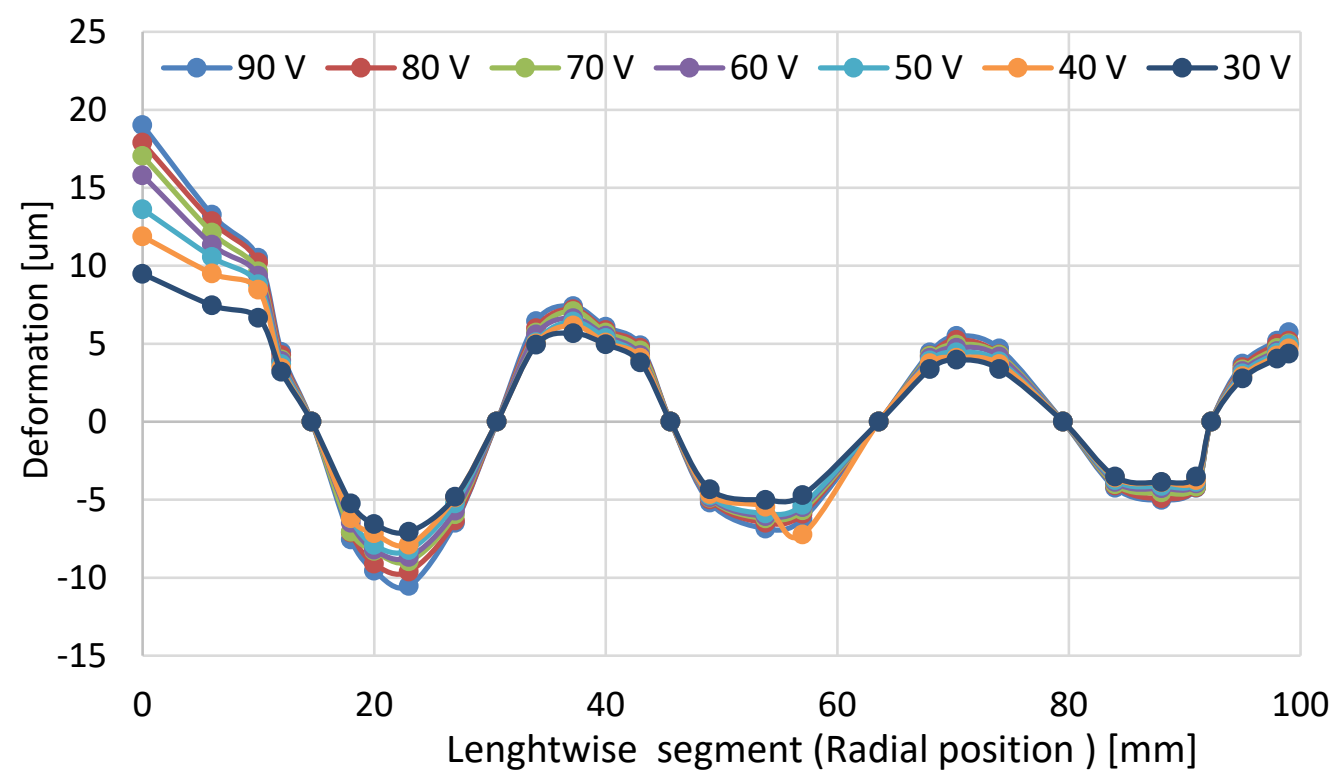

Fig. 9 Experimental dynamic disc deformation (Design A1) at resonance frequency of $26.4 \mathrm{kHz}$.

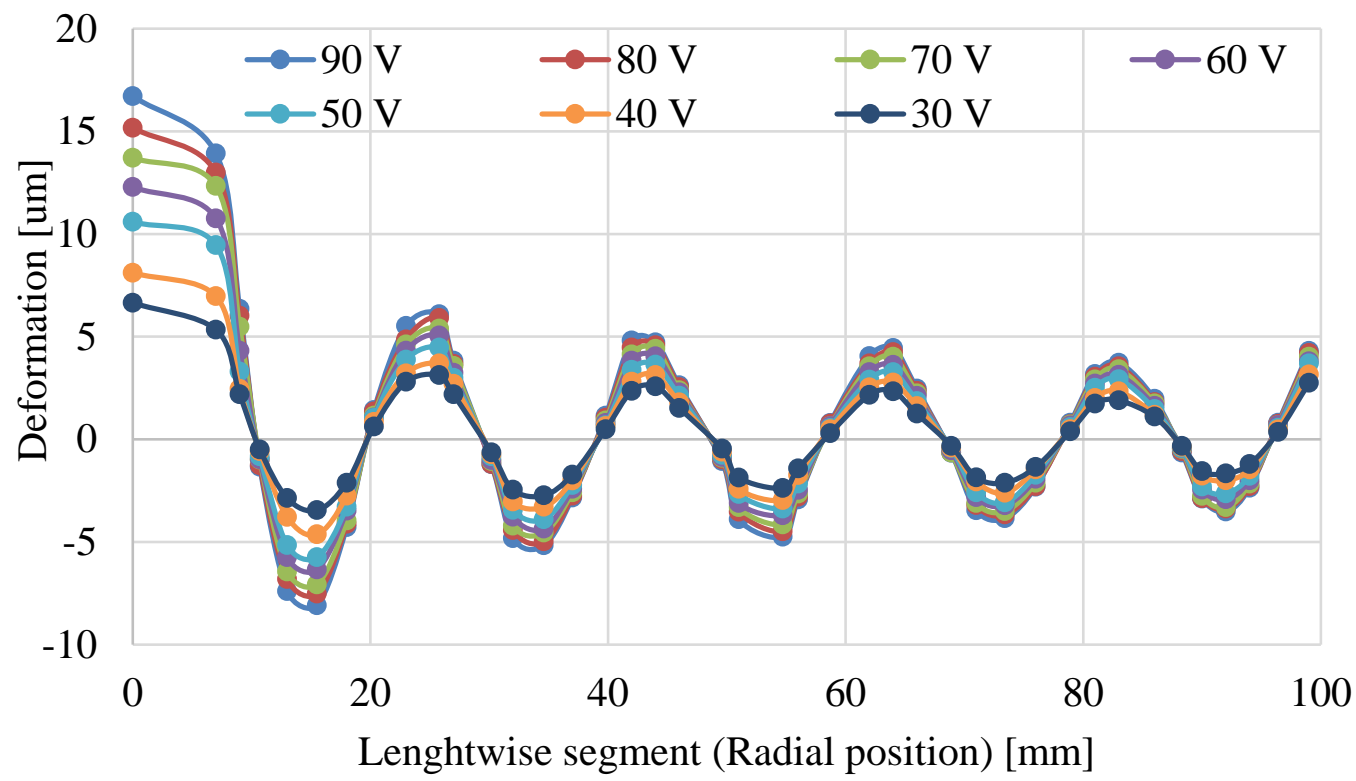

Fig. 10 Experimental dynamic disc deformation (Design A2) at resonance frequency of $25.8 \mathrm{kHz}$. 
As can be seen, the dynamic deformations of designs A1 and A2 are at a maximum at the centre of the disc (Fig.9 and Fig.10) and then start fluctuating at various points of the measured segment. This fluctuation is a result of the plate modal shapes. The deformations of Design A1 and A2 exhibit only a small change when driven at different voltages. This is because the properties of piezoelectric actuators (Langevin type) are less sensitive for a small variation of the driving voltages. The measurements reveal that the maximum dynamic deformation of the disc of Design A1 is about $20 \mu \mathrm{m}$, whereas it is about $17 \mu \mathrm{m}$ for Design A2 at a driving voltage of $90 \mathrm{~V}$. The measurements disclose that the minimum dynamic deformation of the disc of Design A1 and Design A2 at a driving voltage of $30 \mathrm{~V}$ are about 2.7 and $4.5 \mu \mathrm{m}$, respectively. The load capacity (in terms of levitation height) of tested designs (A1 and A2) is shown in Figures 11 and 12 respectively.

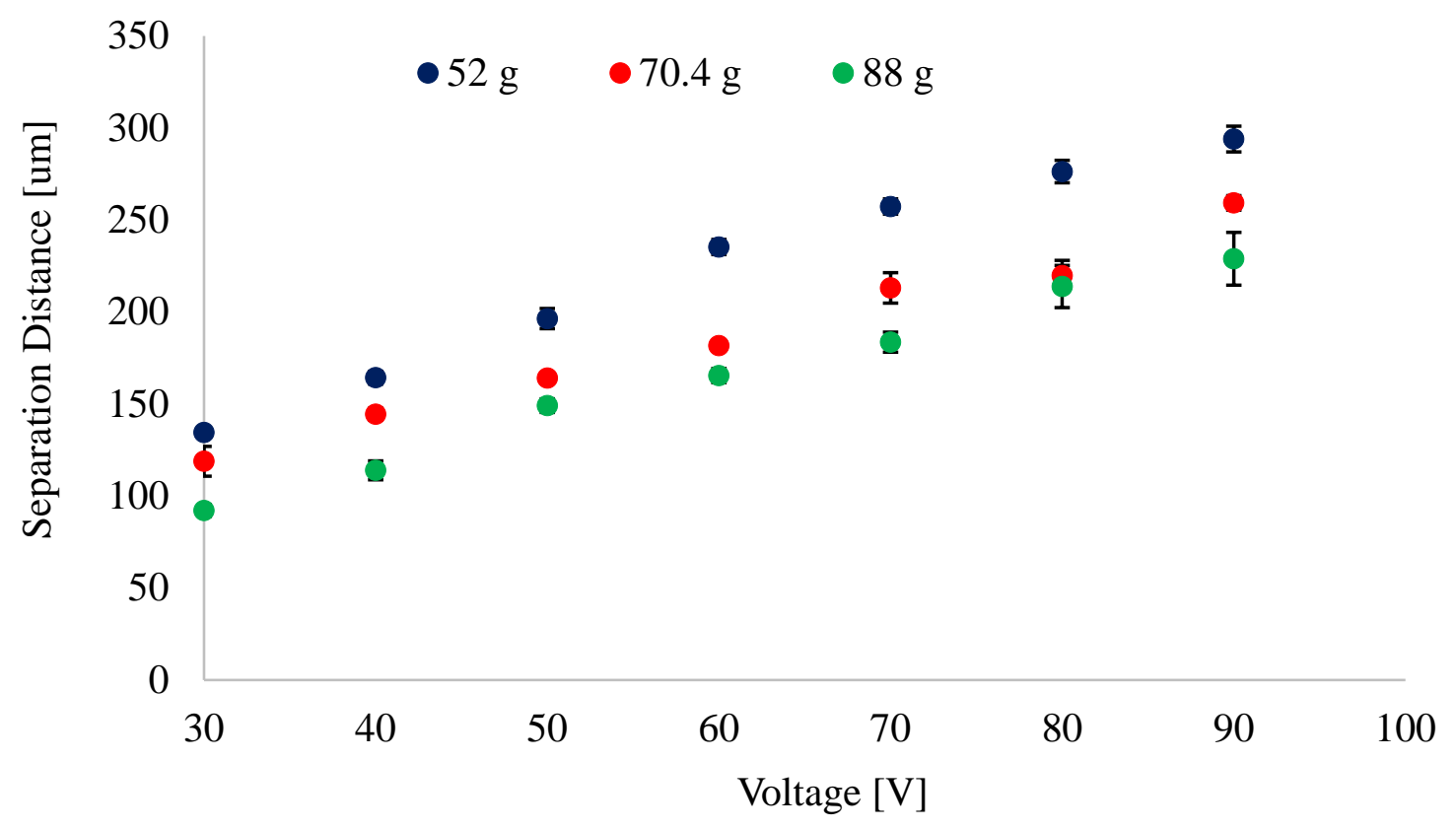

Fig. 11 Experimental separation distance for various weights (wafer) at different driving voltages for Design A1. 


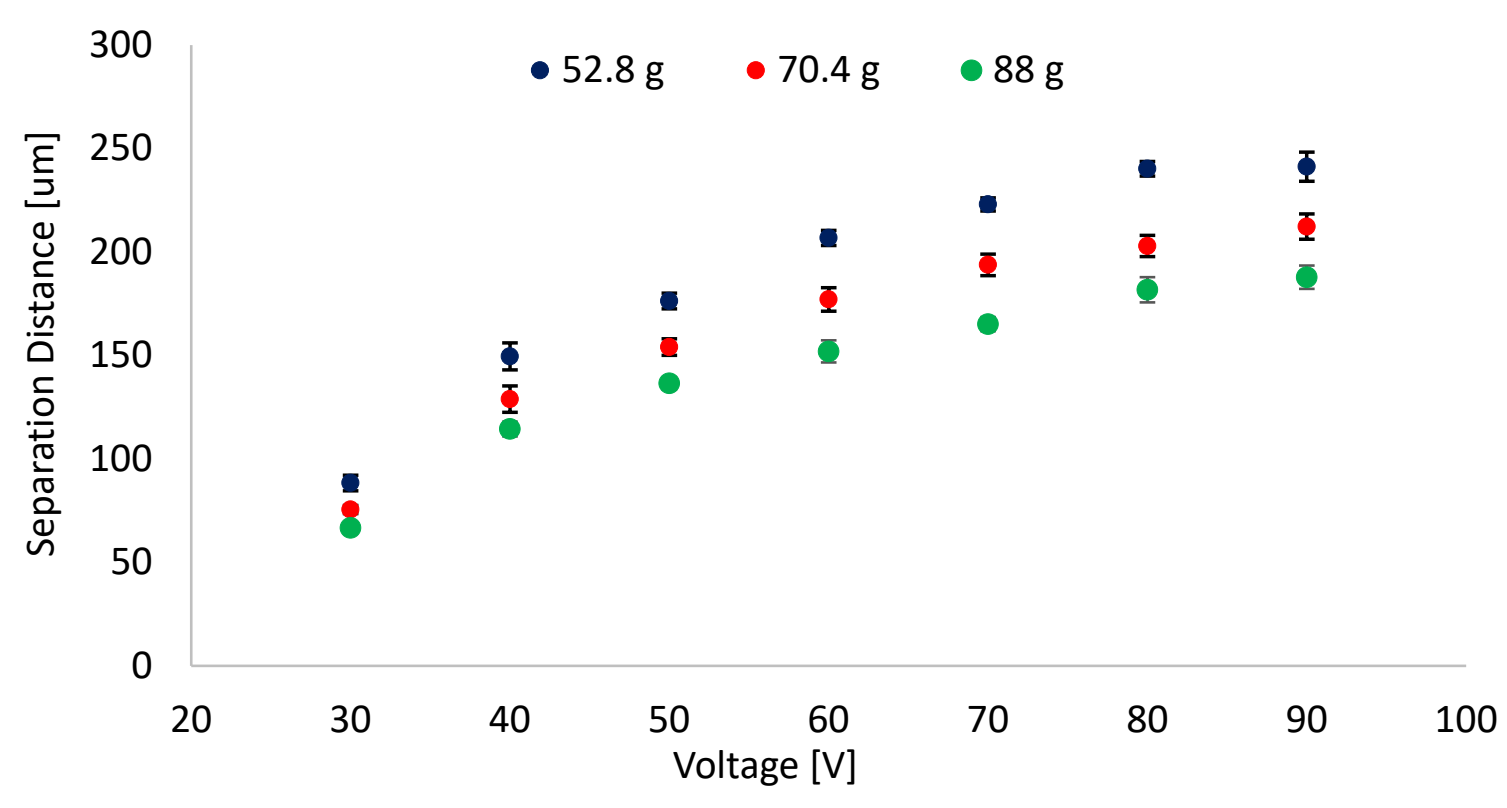

Fig.12 Experimental separation distance for various weights (wafer) at different driving voltages for Design A2.

It can be observed that the relationship between load carrying capacity and the applied voltage is basically linear. Design A1 achieved a higher levitation than Design A2, because the thickness of the disc of Design A1 is $1 \mathrm{~mm}$, whilst for the Design A2 disc it is $2 \mathrm{~mm}$. The highest floating happened for the object of weight 52 g placed on Design A1 at a driving voltage of 90 V, which reached height of about $294 \mu \mathrm{m}$ (see Fig.11), whereas the lowest floating altitude occurred for the object of weight 88 g placed on Design A2 at a driving voltage of 30 [V], which reached elevation of about $66 \mu \mathrm{m}$ (see Fig.12).

\subsection{Design B}

The plate modal shapes and dynamic deformation of the plate's centre point and lengthwise segment have been recorded with the help of the DIC system. This is shown in Figure 13. The plate's behaviour shows that there is a symmetry at its central point and the maximum dynamic deformation of approximately $7 \mu \mathrm{m}$ occurred there due to the constraints imposed on the plate. 

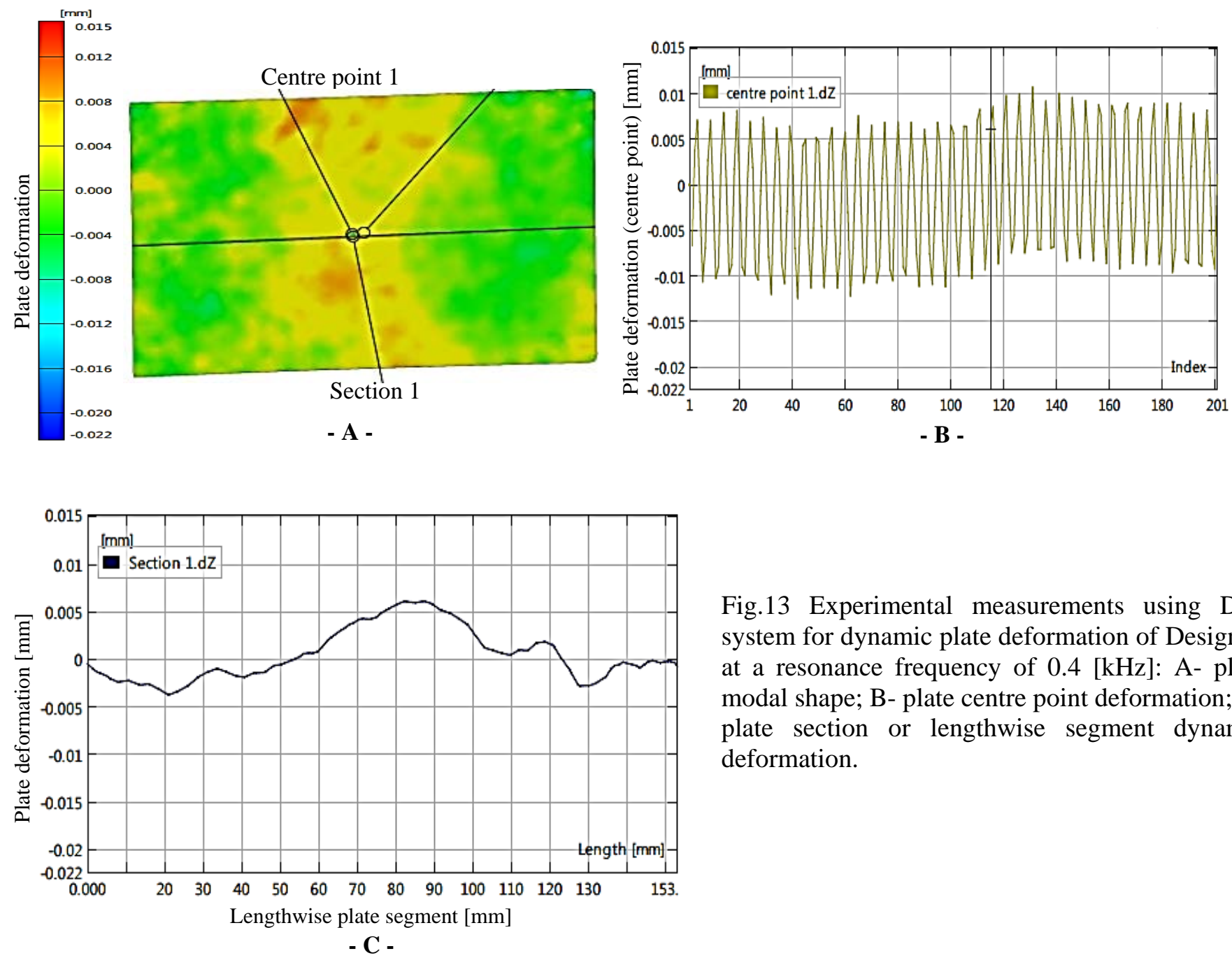

Fig.13 Experimental measurements using DIC system for dynamic plate deformation of Design B at a resonance frequency of $0.4[\mathrm{kHz}]$ : A- plate modal shape; B- plate centre point deformation; Cplate section or lengthwise segment dynamic deformation.

The results for Design B confirm that a weight can be floated at one of the resonant frequencies of the design which is close from $0.4 \mathrm{kHz}$. Driving the system at resonant frequency results in a better levitation performance.

The average value of the plate centre point deformation (Fig. 13-B) tends to change, it could be due to the adhesive used to attach the PZT to the plate. Also, noise from the voltage amplifier and the signal generator can influence the result. Figure 14 illustrates the Design B and the levitated disc with three points on its top surface. The dynamic levitation distance (see Fig. 15) of the three points on the top surface of the disc, which are selected arbitrarily, show that floating of the disc is unstable. The modal shape of the oscillating plate and consequently the distribution of the squeeze-film pressure that is developed underneath the floating disc can cause this trembling floating [19]. The maximum and minimum floating heights for a $5 \mathrm{~g}$ disc floated on 
Design B reach to about 100 and $80 \mu \mathrm{m}$ respectively, whereas the average separation distance is equal to approximately $70 \mu \mathrm{m}$.

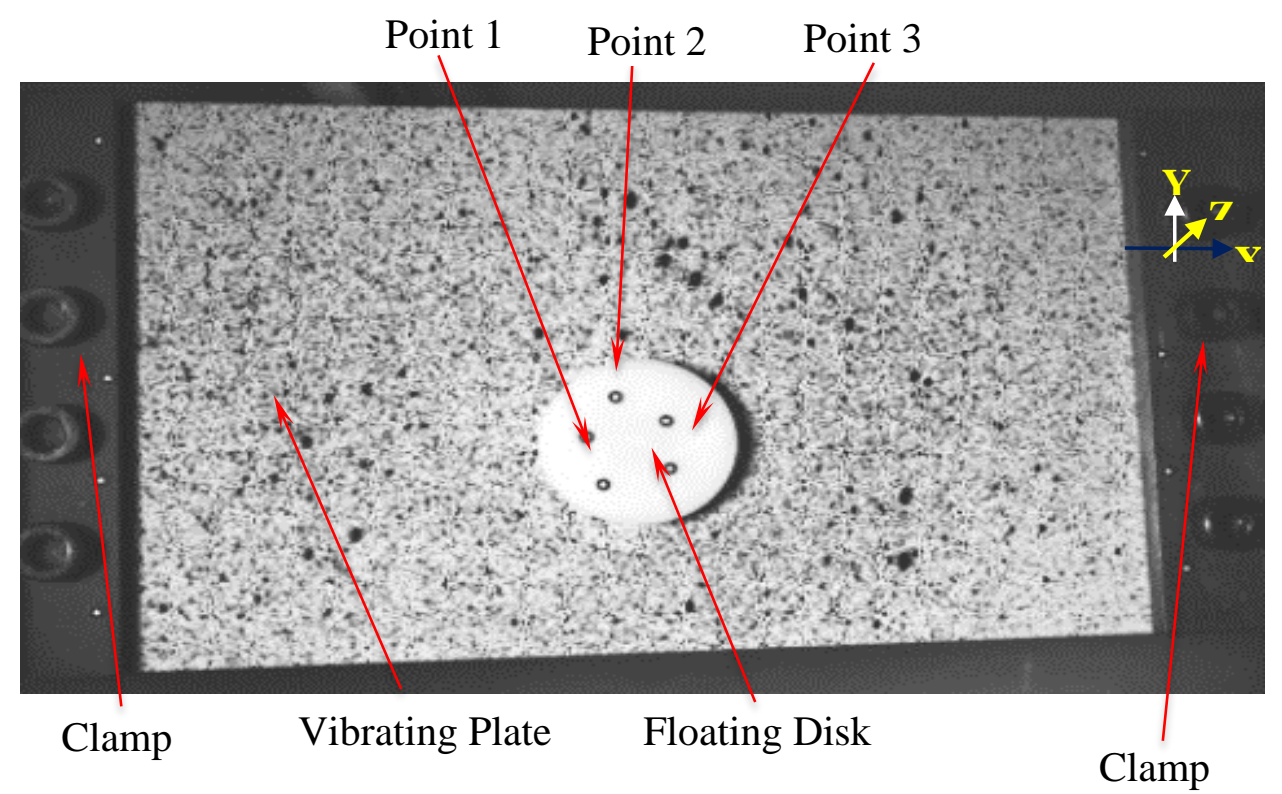

Fig.14 Design B, floating disc under DIC system.

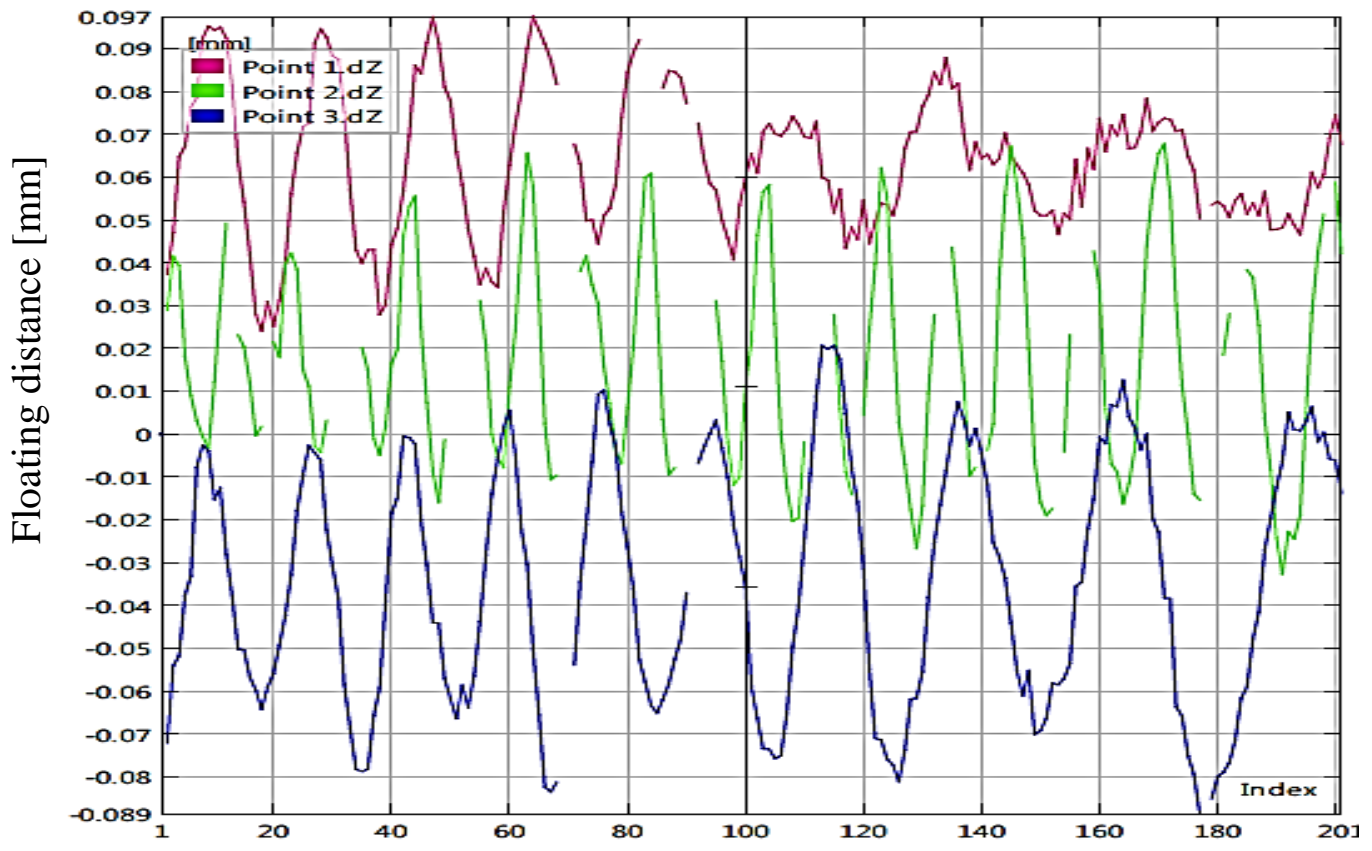

Fig. 15 Separation distance for a disc of 5 [g] floating on Design B driven at $399 \mathrm{~Hz}$. 


\section{Conclusions}

The study has involved investigating two different SFL device designs that could be used for contactless transportation. The main goal was to compare the designs in terms of load carrying capacity and levitation altitude. Experimental investigations have been carried out for both designs to explore their performance. Clearly, the design configuration is important, and, the plate size and geometry as well as the driving boundary conditions. The experimental results highlight that the Design A provides more non-contact lift capabilities than Design B. However, the former is more complex than the latter in terms of its manufacturing. Design A has dynamic characteristics very different to Design B, as Design A has an essential longitudinal modal shape to float the object. Moreover, Design A can create levitation for the object based on different model shapes, for example, bending or flexural. In addition, Design A is not constrained whereby the plate (Design B) is clamped at its both ends. Hence, it has more free edges that leads to a positive impact on pressure distribution and accordingly, on levitation behaviour. In contrast, Design B is clamped at the ends, which has a more complex effect on modal shapes, distribution of the squeeze-film pressure underneath the floating object and consequently, on its floating performance.

Moreover, the dynamic deformation of the disc of Design A is amplified two times, initially, through the voltage amplifier and secondly, by the horn geometry.

In contrast, the dynamic deformation of the plate in Design B is magnified only through the voltage amplifier equipment. It can be concluded that Design A is better than Design B in terms of load carrying capacity. However, Design A is more complicated in terms of design and manufacturing, so it is costlier than Design B. In addition, repairing Design A is intricate unlike design B which is simple to replace a defective actuator. Finally, Design B is more suitable to be utilised as a linear conveyor. 


\section{Acknowledgements}

One of the authors (AA) would like to acknowledge the scholarship of the Iraqi Ministry of Higher Education and Scientific Research, Al-Furat Al-Awsat Technical University/Najaf/Iraq and the GOM Company.

\section{References}

[1] Stolarski, T., A., and Miyatake, M., 2018. Acoustic Journal Bearing with Changeable Geometry and Built-in Flexibility, ASME Journal of Tribology.

[2] Langlois, W. E., 1962. Isothermal squeeze films. Quarterly of Applied Mathematics, Vol. XX, No. 2, pp.131-150.

[3] Hashimoto, Y., Yoshikazu, K. \& Sadayuki, U., 1996. Near - field acoustic levitation of planar specimens using flexural vibration. The Journal of the Acoustical Society of America, 100(4), pp. 2057-2061.

[4] Matsuo, E., Koike, Y., Nakamura, K., Ueha, S. and Hashimoto, Y., 2000. Holding characteristics of planar objects suspended by near-field acoustic levitation. Ultrasonics, 38(1-8), pp.60-63.

[5] Ueha, S., Yoshiki, H. \& Yoshikazu, K., 2000. Non-contact transportation using near-field acoustic levitation. Ultrasonic, 38(1-8), pp. 26-32.

[6] Minikes, A. and Bucher, I., 2003. Coupled dynamics of a squeeze-film levitated mass and a vibrating piezoelectric disc: numerical analysis and experimental study. Journal of Sound and Vibration, 263(2), pp.241-268.

[7] Nomura, H., Kamakura, T. and Matsuda, K., 2002. Theoretical and experimental examination of nearfield acoustic levitation. The Journal of the Acoustical Society of America, 111(4), pp.1578-1583.

[8] Hu, J., Nakamura, K. and Ueha, S., 2003. Stability analysis of an acoustically levitated disk. IEEE transactions on ultrasonic, ferroelectrics, and frequency control, 50(2), pp.117-127.

[9] Atherton, M.A., Mares, C. and Stolarski, T. A., 2014. Some fundamental aspects of self-levitating sliding contact bearings and their practical implementations. Proceedings of the Institution of Mechanical Engineers, Part J: Journal of Engineering Tribology, 228(9), pp.916-927.

[10] Minikes, A., Bucher, I. and Haber, S., 2004. Levitation force induced by pressure radiation in gas squeeze films. The Journal of the Acoustical Society of America, 116(1), pp.217-226. 
[11] Stolarski, T. \& Woolliscroft, C., 2007. Use of near-field acoustic levitation in experimental sliding contact. Journal of applied mechanics, 74(4), pp. 816-820.

[12] Wang, Y. and Wei, B., 2013. Mixed-modal disk gas squeeze film theoretical and experimental analysis. International Journal of Modern Physics B, 27(25), p.1350168.

[13] Ilssar, D. and Bucher, I., 2015. On the slow dynamics of near-field acoustically levitated objects under High excitation frequencies. Journal of Sound and Vibration, 354, pp.154-166.

[14] Ilssar, D., Bucher, I. \& Cohen, N., 2014. Structural optimization for one dimensional acoustic levitation devices - Numerical and experimental study. USA.

[15] Chang, X., Wei, B., Atherton, M., Mares, C., Stolarski, T. and Almurshedi, A., 2016. NFAL prototype design and feasibility analysis for self-levitated conveying. Tribology Transactions, 59(5), pp.957-968.

[16] Wei, B., Shaham, R. and Bucher, I., 2018. Theoretical investigation and prototype design for nonparallel squeeze film movement platform driven by standing waves. Tribology International, 119, pp.539548.

[17] Li, W., Yuanyuan, L. \& Feng, K., 2017. Modelling and experimental study on the influence of surface grooves on near-field acoustic levitation. Tribology International, Volume 116, pp. 138-146.

[18] Vandaele, V., Lambert, P. and Delchambre, A., 2005. Non-contact handling in micro assembly: Acoustical levitation. Precision engineering, 29(4), pp.491-505.

[19] Kim, C. H. and Ih, J. G., 2007. On the horizontal wobbling of an object levitated by near-field acoustic levitation. Ultrasonic, 46(4), pp.331-335.

[20] Liu, P., Li, J., Ding, H. and Cao, W., 2009. Modeling and experimental study on near-field acoustic levitation by flexural mode. IEEE transactions on ultrasonics, ferroelectrics, and frequency control, $56(12)$. 